\title{
Interaction Effects at Crossings of Spin-Polarized One-Dimensional Subbands
}

\author{
A. C. Graham, K. J. Thomas, M. Pepper, N. R. Cooper, M. Y. Simmons, ${ }^{*}$ and D. A. Ritchie \\ Cavendish Laboratory, Madingley Road, Cambridge, CB3 OHE, United Kingdom
}

(Received 7 May 2003; published 25 September 2003)

\begin{abstract}
We report conductance measurements of ballistic one-dimensional (1D) wires defined in GaAs/ AlGaAs heterostructures in an in-plane magnetic field, $B$. When the Zeeman energy is equal to the 1D subband energy spacing, the spin-split subband $N \uparrow$ intersects $(N+1) \downarrow$, where $N$ is the index of the spin-degenerate $1 \mathrm{D}$ subband. At the crossing of $N=1 \uparrow$ and $N=2 \downarrow$ subbands, there is a spontaneous splitting giving rise to an additional conductance structure evolving from the $1.5\left(2 e^{2} / h\right)$ plateau. With further increase in $B$, the structure develops into a plateau and lowers to $2 e^{2} / h$. With increasing temperature and magnetic field the structure shows characteristics of the 0.7 structure. Our results suggest that at low densities a spontaneous spin splitting occurs whenever two 1D subbands of opposite spins cross.
\end{abstract}

DOI: 10.1103/PhysRevLett.91.136404

Studies of ballistic transport in one dimension (1D) have shown that a spontaneous spin splitting may occur at zero magnetic field, as indicated by a conductance structure at $0.7\left(2 e^{2} / h\right)$, which drops to $0.5\left(2 e^{2} / h\right)$ in an inplane magnetic field [1]. This so-called 0.7 structure is widely reported in various types of ballistic $1 \mathrm{D}$ wires defined in GaAs [1-4] and Si heterostructures [5]. In some cases, at very low electron densities a structure has been observed at $0.5\left(2 e^{2} / h\right)$ at zero magnetic field which strengthened with in-plane magnetic field [6], indicating a complete spin polarization [7,8].

By studying Zeeman splitting of 1D subbands, it was shown that the 0.7 structure is accompanied by an enhancement of the Lande $g$ value as the 1D subbands are depopulated, and the energy difference between the spinsplit 1D subbands tends to a finite value at zero magnetic field [1]. The conductance plateau at $0.5\left(2 e^{2} / h\right)$, either observed in zero magnetic field or induced by Zeeman effect, rises to $0.6\left(2 e^{2} / h\right)$ with increasing temperature [9]. None of these characteristics can be explained within a single particle model. As the situation is dynamic, for short ballistic 1D wires, zero-field spin splitting may not be in conflict with the theorem of Lieb and Mattis [10].

The discovery of the 0.7 structure in ballistic $1 \mathrm{D}$ wires has stimulated much theoretical work in one dimension, some of which focused on zero-field spin polarization [11-13], spin density wave formation [14], pairing of electrons [15], singlet-triplet formation [16], Kondo-like interactions $[4,17,18]$, and electron-phonon effects [19]. Experimental studies [1-3] of the 0.7 structure have, in general, indicated that due to a spin splitting at zero magnetic field, a complex many-body state may exist in a ballistic 1D constriction. In order to further study the role of spin, a strong magnetic field is applied parallel to a quantum wire to produce large Zeeman splitting and induce crossings between spin-split 1D subbands [20]. In this Letter, we show that at the crossing of Zeemansplit 1D subbands of opposite spins and different spatial
PACS numbers: 71.70.-d, 72.25.Dc, 73.21.Hb, 73.23.Ad

wave functions, a spontaneous splitting sets in, giving rise to new conductance structures exhibiting characteristics of the 0.7 structure; we call this new structure a 0.7 analog. We have observed such 0.7 analogs in eight samples, with magnetic field applied in both in-plane directions.

Split-gate devices were defined by electron beam lithography on a Hall bar etched from a high mobility GaAs $/ \mathrm{Al}_{x} \mathrm{Ga}_{1-x} \mathrm{As}$ heterostructure. Samples A and B used in this work have a length $0.4 \mu \mathrm{m}$ and widths 0.6 and $0.5 \mu \mathrm{m}$. The two-dimensional electron gas (2DEG) formed $292 \mathrm{~nm}$ below the surface has a mobility of $1.1 \times$ $10^{6} \mathrm{~cm}^{2} / \mathrm{Vs}$ and a carrier density of $1.15 \times 10^{11} \mathrm{~cm}^{-2}$. Conductance measurements were performed in a dilution refrigerator using an excitation voltage of $10 \mu \mathrm{V}$ at $77 \mathrm{~Hz}$. The samples were mounted with the magnetic field, $B_{\|}$, parallel to the current direction. By monitoring the Hall voltage, the out-of-plane misalignment was measured to be less than $0.5^{\circ}$.

Figure 1 shows differential conductance $G=\frac{d I}{d V}$ traces, measured as a function of split-gate voltage $V_{g}$ at fixed magnetic fields, $B_{\|}$. The inset shows a schematic illustration of linear Zeeman splitting of 1D energy subbands for a parabolic potential confinement. The left trace of the main figure shows conductance plateaus quantized at $N\left(2 e^{2} / h\right)$ and the 0.7 structure at $B_{\|}=0$. As $B_{\|}$is incremented to $15.6 \mathrm{~T}$ (right trace), the overall conductance characteristics undergo three major changes. These correspond to $\mathrm{P}, \mathrm{Q}$ and $\mathrm{R}$ in Fig. 1 inset. First, each spindegenerate 1D subband $N$ splits into two, $N \uparrow$ and $N \downarrow$ (see $\mathrm{P}$ in the inset), and new conductance plateaus appear at half-integer values of $2 e^{2} / h$. Second, with further increase of $B_{\|}$, the half-integer plateaus strengthen and integer plateaus weaken. When the Zeeman energy, $g \mu_{B} B_{\|}$, is equal to the subband energy spacing, $\Delta E_{N, N+1}$, integer plateaus disappear. This happens when the split levels, for example, $N \uparrow$ and $(N+1) \downarrow$ converge and pass through a crossing point (see $\mathrm{Q}$ in the inset). 


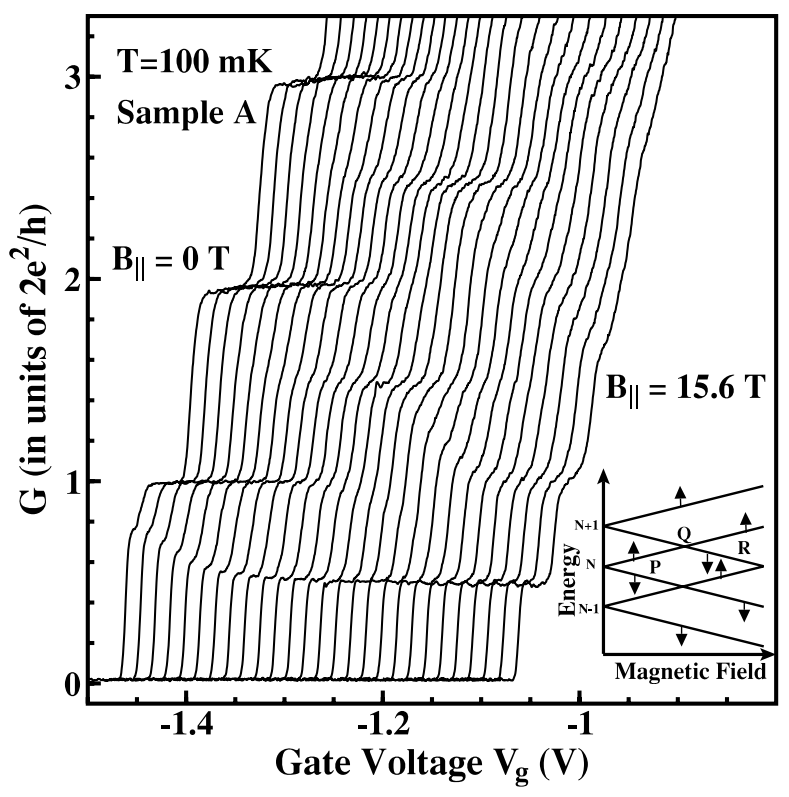

FIG. 1. Differential conductance, $G\left(V_{g}\right)$, traces at $B_{\|}$incremented in steps of 0.6 T. For clarity, successive traces are offset horizontally. Inset: Schematic energy diagram for a linear Zeeman splitting of 1D subbands and subsequent crossings.

Finally, with further increase of $B_{\|}$, half-integer plateaus weaken and integer plateaus reappear as the $N \uparrow$ and $(N+$ $1) \downarrow$ diverge again after the crossing (see $R$ in the inset). For example, the plateau at $1.5\left(2 e^{2} / h\right)$ weakens and the $2 e^{2} / h$ plateau reappears for $B_{\|}>8 \mathrm{~T}$. However, this is accompanied by the evolution of a weak structure from the edge of the $1.5\left(2 e^{2} / h\right)$ plateau, which gradually lowers to $2 e^{2} / h$ and develops into a plateau. This resembles the evolution of the 0.7 structure to $0.5\left(2 e^{2} / h\right)$ with increasing $B_{\|}$. For this reason, we call the structure at the crossing a 0.7 analog. It may be noted that the reappearing $2 e^{2} / h$ plateau now carries the opposite spin to that before crossing. The lowest subband $1 \downarrow$, however, does not encounter a crossing; therefore the plateau at $0.5\left(2 e^{2} / h\right)$ is intact, and does not change its spin.

The evolution of conductance characteristics with the splitting of $1 \mathrm{D}$ subbands in $B_{\|}$can be clearly represented in a gray-scale plot of the transconductance $d G / d V_{g}$, obtained by numerical differentiation of $G\left(V_{g}\right)$ characteristics. Figure 2(a) shows $d G / d V_{g}$ plots of sample B as a function of $B_{\|}$and $V_{g}$. White regions represent low transconductance [plateaus in $G\left(V_{g}\right)$ ] and the dark thick lines correspond to high transconductance (transitions between plateaus). Each dark line splits into two as $B_{\|}$increases. This can be interpreted as the splitting of each 1D subband into two subbands of opposite spins as shown by $\mathrm{P}$ in the Fig. 1, inset. On the left of Fig. 2(a), for $N=1$, there are two distinct dark lines at $B_{\|}=0$. The white region between these two dark lines represents the 0.7 structure, marked by $\alpha_{0}$. As the gap between the $N=1 \downarrow$ and $N=$ $1 \uparrow$ widens with $B_{\|}$, the 0.7 structure evolves into a plateau

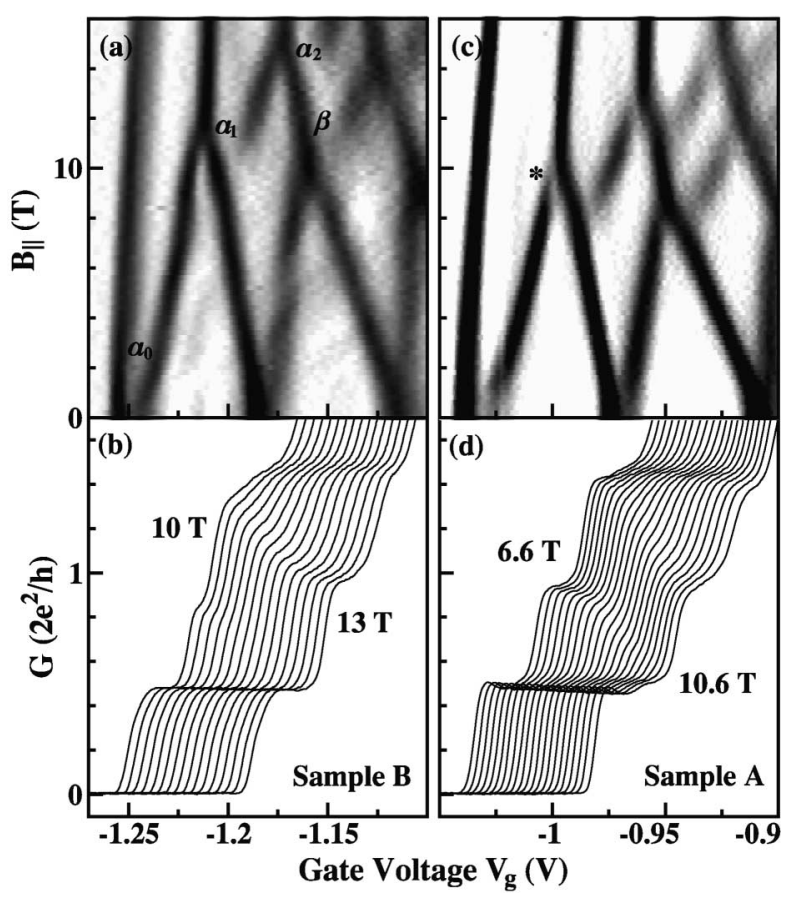

FIG. 2. (a) Gray-scale plot of transconductance, $d G / d V_{g}$, as a function of $V_{g}$ and $B_{\|}$for sample B. (b) $G\left(V_{g}\right)$ traces (offset horizontally) for $B_{\|}=10 \mathrm{~T}$ to $13 \mathrm{~T}$ incremented by $0.2 \mathrm{~T}$ (sample B, different cooldown). (c) gray-scale plot as in (a), for sample A. (d) $G\left(V_{g}\right)$ traces as in (b), for sample A.

at $0.5\left(2 e^{2} / h\right)$ and the white region $\left(\alpha_{0}\right)$ in Fig. 2(a) broadens. At $B_{\|} \approx 11 \mathrm{~T}$, dark lines corresponding to $N=1 \uparrow$ and $N=2 \downarrow$ subbands cross. After the crossing, the line $N=1 \uparrow$ shows a discontinuous shift of $\delta V_{g}=23 \mathrm{mV}$ from the crossing point, marked by $\alpha_{1}$. This discontinuity in the dark line $N=1 \uparrow$ corresponds to the appearance of the 0.7 analog. Figure 2(b) shows $G\left(V_{g}\right)$ traces in the vicinity of $\alpha_{1}$ from a different cooldown of the sample, highlighting the evolution of the 0.7 analog from the edge of $1.5\left(2 e^{2} / h\right)$ plateau to $2 e^{2} / h$ with increasing $B_{\|}$. It is observed that the $1.5\left(2 e^{2} / h\right)$ plateau, though weakening, remains visible when the 0.7 analog evolves.

The discontinuous evolution of the right-moving dark lines ( $\uparrow$-spin subbands) can also be observed at the crossing of $N=2 \uparrow$ with $N=3 \downarrow$ lines marked by $\beta$, and at the second crossing of $N=1 \uparrow$ with $N=3 \downarrow$ line, marked by $\alpha_{2}$. Figures 2(c) and 2(d) show results of sample $\mathrm{A}$ in a different cooldown from that of Fig. 1. In addition to the splittings at the crossing of peaks as observed in sample $\mathrm{B}$, in this case there is also a splitting just before the crossing of $N=1 \uparrow$ and $N=2 \downarrow$ peaks, marked by an asterisk in Fig. 2(c). In one of the cooldowns, sample B also showed a weak splitting before the crossing. It is not clear whether this splitting marked by asterisk is related to $\alpha_{1}$, the 0.7 analog. It may be observed that this corresponds to a slower rate of suppression of the $2 e^{2} / h$ plateau (1 $\uparrow$-subband) in sample A with $B_{\|}$as shown in Fig. 2(d), compared to sample B in Fig. 2(b). 
In the following, we suggest that the main features of our observations arise from strong electron-electron interactions. To clarify this, we first describe the expected behavior in the case of noninteracting electrons. In particular, we show that the diamagnetic shifts of the subband energies in an in-plane magnetic field have only a very limited influence on the most important features of our observations.

Figure 3(a) shows a gray-scale plot of sample B with many occupied 1D subbands, part of which is shown in Fig. 2(a). Figure 3(b) shows the positions of the calculated transconductance peaks as a function of electron density and $B_{\|}$for a model of noninteracting electrons in an infinite 1D wire. This model includes the diamagnetic effects with $B_{\|}$, assuming parabolic confinement in the transverse and vertical (quantum well) directions with subband spacings of 1.85 and $15 \mathrm{meV}$ [21], respectively. A $g$ value of 1.9 is used in this model in accordance with the value measured at low $B_{\|}$in our samples.

If one assumes that the electron density in the wire is linearly related to $V_{g}$, then Figs. 3(a) and 3(b) can be compared directly. Clearly the model of noninteracting electrons accounts well for the general trends in the
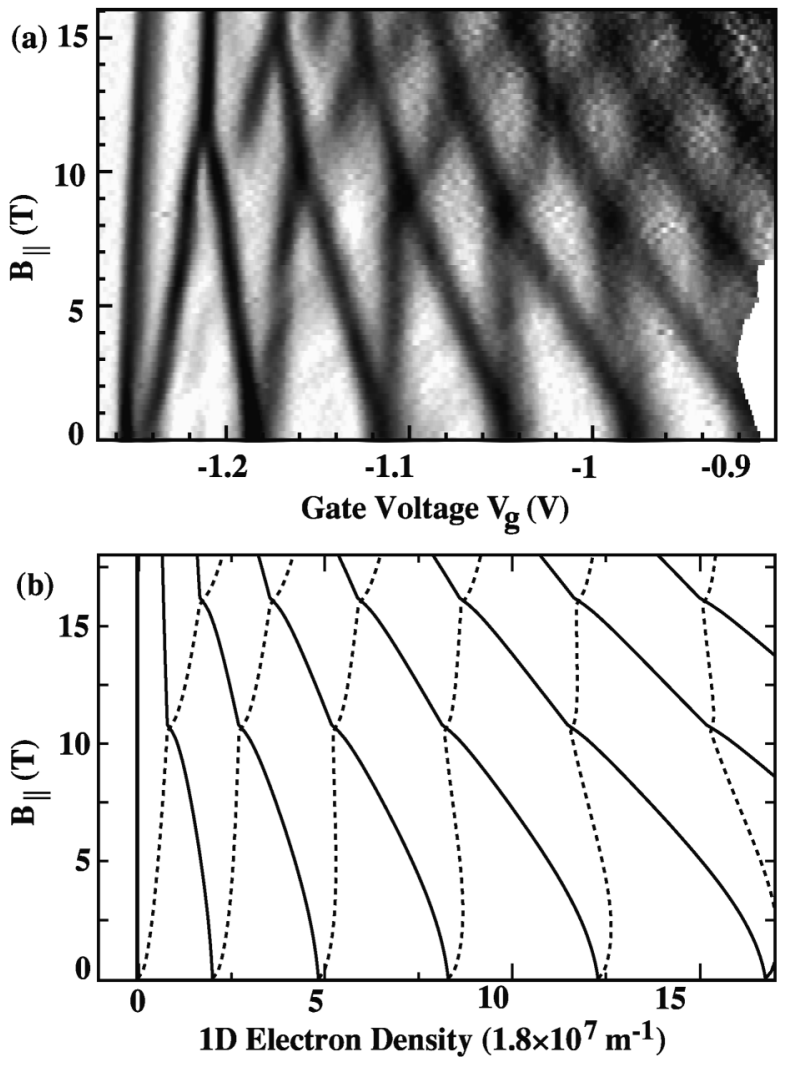

FIG. 3. (a) Gray-scale plot of $d G / d V_{g}$ as a function of $V_{g}$ and $B_{\|}$for sample $\mathrm{B}$, showing the splitting of five 1D subbands. (b) Calculated Zeeman splitting with diamagnetic shift of the 1D subbands. The solid lines represent parallel spin and the dotted lines represent antiparallel spin. evolution of the transconductance peaks with $V_{g}$ and $B_{\|}$. However, the model cannot capture the appearance of discontinuities in the positions of the transconducance peaks at the crossings, $\alpha_{1}, \alpha_{2}$, and $\beta$ in Fig. 2. As we have described above, these are the regions where the conductance displays the 0.7 analogs.

Figure 4 shows the temperature dependence of the 0.7 analog at the first crossing of $1 \uparrow$ and $2 \downarrow$ subbands. A defining characteristic of the 0.7 structure is its unusual temperature dependence. In addition, at low temperatures, the 0.7 structure becomes well defined only at low electron densities [6]. For a higher 1D density, a higher temperature (typically, $T \approx 1 \mathrm{~K}$ in GaAs split-gate devices [1]) is required to induce the 0.7 structure [2]. At high $B_{\|}$, it is well known that the 0.7 structure develops into a plateau at $0.5\left(2 e^{2} / h\right)$; however, with an increase in $T$, the plateau rises to $0.6\left(2 e^{2} / h\right)$ [9]. These trends in the temperature dependence of the 0.7 structure and $0.5\left(2 e^{2} / h\right)$ plateau are also observed in the new 0.7 analog structure at $B_{\|}=10 \mathrm{~T}$ and $2 e^{2} / h$ plateau at $B_{\|}=13 \mathrm{~T}$. Figure 4 shows that, at $B_{\|}=10 \mathrm{~T}$, the 0.7 analog present just below $1.5\left(2 e^{2} / h\right)$ (shown by a down arrow) drops as $T$ rises; but at $B_{\|}=13 \mathrm{~T}$, the plateau at $2 e^{2} / h$ (shown by an up arrow) rises with increasing $T$. In the inset of Fig. 4, conductance of the 0.7 analog is plotted as a function of $B_{\|}$for a range of temperatures. These data compare well to the temperature dependence of the 0.7 structure [9], showing the crossover of $G$ as a function of $T$ at a characteristic $B_{\|}$.

Nonquantized conductance structures can be due to a change in the transmission probability caused by scattering or a many-body effect in the 1D channel. We have observed 0.7 analogs in eight samples, and they are

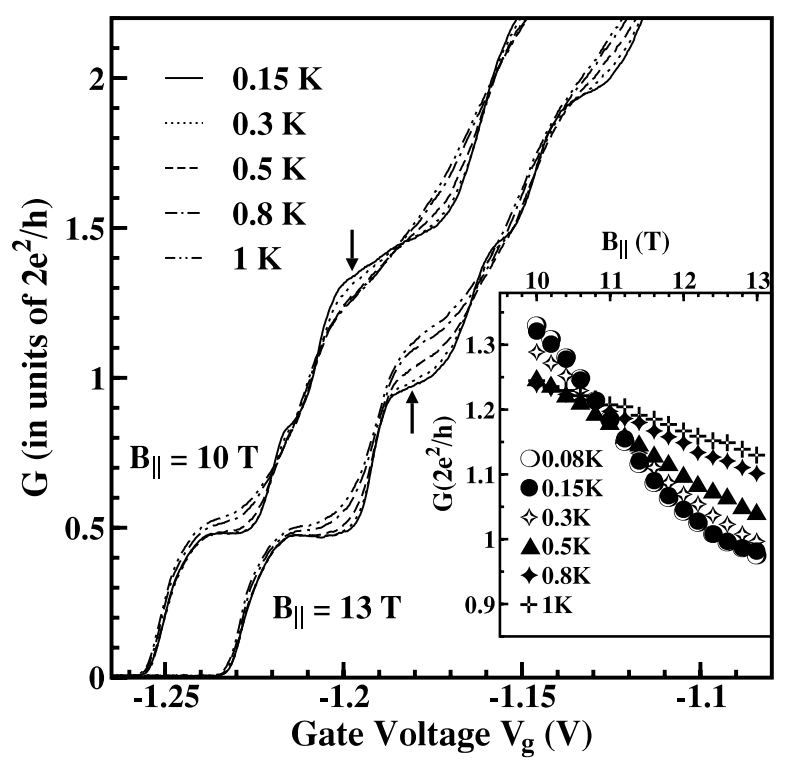

FIG. 4. Temperature dependence of $G\left(V_{g}\right)$ for sample B [same cooldown as Fig. 2(b)] at 10 and 13 T. Inset: The height of the 0.7 analog as a function of $B_{\|}$for various temperatures. 
independent of cooldowns, and occur only at the crossing of spin-split subbands of opposite spins. Because of the high reproducibility of the 0.7 analogs, a disorder-induced scattering effect can be discounted.

When two energy levels are brought together, an anticrossing may occur. However, this depends on the symmetry of the two wave functions. In our case, the two 1D levels that cross in $B_{\|}$have different spins and subband indices; therefore such anticrossings should be very weak. Experimentally, we do not observe anticrossings of 1D subbands, rather a gap forms abruptly after the crossing. As in the case of the 0.7 structure, we believe that the new 0.7 analog is a consequence of strong exchange interactions. In the former case, there is a lifting of the zero-field spin degeneracy, whereas in the latter case, the degeneracy at the crossing point is lifted. We can quantify the strength of the exchange interactions by measuring the gate voltage splitting at $\alpha_{1}$. From dc source-drain bias calibration [22], $\alpha_{1}$ is measured to be $0.5 \mathrm{meV}$, which is a third of the subband spacing $\left(\Delta E_{1,2}=1.6 \mathrm{meV}\right)$ at zero magnetic field.

Given the strong similarity between the 0.7 analog and the 0.7 structure, we consider whether theories for the 0.7 structure could apply to our results. A recently proposed electron-phonon scattering mechanism for the 0.7 structure [19] cannot account for the observed 0.7 analog. Consider Fig. 2(b): if the strong 0.7 analog in these traces were the result of a conductance suppression caused by electron-phonon scattering, then one should expect at least as strong a suppression at the spin-polarized $0.5\left(2 e^{2} / h\right)$ plateau; there is no such indication.

The behavior of the 0.7 analog does not seem to be consistent with the most simple extension of a proposed "Kondo model" for the 0.7 structure [17] to the present situation - in which a Kondo impurity forms from quasibound states of the $1 \uparrow$ and $2 \downarrow$ levels which become degenerate at some nonzero $B_{\|}$. In this model, one would expect the 0.7 analog feature to fall onto the $2 e^{2} / h$ plateau as $B_{\|}$is increased or decreased away from the point of degeneracy in either direction. In contrast, the 0.7 analog evolves asymmetrically about the midpoint of the crossing. A detailed analysis within the Kondo model will be considered in a later publication.

To conclude, we have observed the crossings of spinsplit 1D subbands of different spins and spatial wave functions in a 1D electron gas. At crossings, there is a spontaneous splitting giving rise to new conductance structures. There are no indications of anticrossings, but an energy splitting may occur due to exchange interactions whenever two 1D subbands are degenerate. The magnetic field and temperature dependences show that the new structures strongly resemble the zerofield 0.7 structure. We believe that these 0.7 analog structures may provide the key to a fuller understanding of the role of electron-electron interactions in ballistic $1 \mathrm{D}$ wires.

We thank J. T. Nicholls, D. Khmelnitskii, V. Tripathi, and C. J. B. Ford for useful discussions. This work was supported by EPSRC, U.K. K. J.T. acknowledges support from the Royal Society.

*Current address: University of New South Wales, School of Physics, Sydney, NSW 2052, Australia.

[1] K. J. Thomas, J. T. Nicholls, M. Y. Simmons, M. Pepper, D. R. Mace, and D. A. Ritchie, Phys. Rev. Lett. 77, 135 (1996).

[2] A. Kristensen et al., Physica B (Amsterdam) 251, 180 (1998).

[3] D. J. Reilly et al., Phys. Rev. B 63, 121311 (2001).

[4] S. M. Cronenwett, H. J. Lynch, D. Goldhaber-Gordon, L. P. Kouwenhoven, C. M. Marcus, K. Hirose, N. S. Wingreen, and V. Umansky, Phys. Rev. Lett. 88, 226805 (2002).

[5] N.T. Bagraev, A. D. Buravlev, L. E. Klyachkin, A. M. Malyarenko, W. Gehlhoff, V. K. Ivanov, and I. A. Shelykh, Semiconductors 36, 439 (2002).

[6] K. J. Thomas, J. T. Nicholls, M. Pepper, W. R. Tribe, M. Y. Simmons, and D. A. Ritchie, Phys. Rev. B 61, 13365 (2000).

[7] C.-K. Wang and K.-F. Berggren, Phys. Rev. B 54, 14257 (1996).

[8] A. Gold and L. Calmels, Philos. Mag. Lett. 74, 33 (1996).

[9] K. J. Thomas, J.T. Nicholls, M. Pepper, M. Y. Simmons, D. R. Mace, and D. A. Ritchie, Physica E (Amsterdam) 12, 708 (2002).

[10] E. Lieb and D. Mattis, Phys. Rev. 125, 164 (1962).

[11] B. Spivak and F. Zhou, Phys. Rev. B 61, 16730 (2000).

[12] H. Bruus, V.V. Cheianov, and K. Flensberg, Physica E (Amsterdam) 10, 97 (2001).

[13] A. A. Starikov, I. I. Yakimenko, and K.-F. Berggren, Phys. Rev. B 67, 235319 (2003).

[14] S. M. Reimann, M. Koskinen, and M. Manninen, Phys. Rev. B 59, 1613 (1999).

[15] V.V. Flambaum and M. Y. Kuchiev, Phys. Rev. B 61, 7869 (2000).

[16] T. Rejec, A. Ramsak, and J. H. Jefferson, Phys. Rev. B 62, 12985 (2000)

[17] Y. Meir, K. Hirose, and N. S. Wingreen, Phys. Rev. Lett. 89, 196802 (2002).

[18] P. E. Lindelof, Proc. SPIE Int. Soc. Opt. Eng. 4415, 12 (2001).

[19] G. Seelig and K. A. Matveev, Phys. Rev. Lett. 90, 176804 (2003).

[20] A. J. Daneshvar, C. J. B. Ford, A. R. Hamilton, M. Y. Simmons, M. Pepper, and D. A. Ritchie, Phys. Rev. B 55, 13409 (1997).

[21] T. Ando, J. Phys. Soc. Jpn. 51, 3893 (1982).

[22] N. K. Patel, J.T. Nicholls, L. Martín-Moreno, M. Pepper, J. E. F. Frost, D. A. Ritchie, and G. A. C. Jones, Phys. Rev. B 44, 13549 (1991). 